\title{
On the Existence of Positive Solutions for Nonlinear Two-point Boundary-value Problems
}

\author{
R. MENNICKEN ${ }^{a}, *$ and D. RACHINSKII ${ }^{b, \dagger}$ \\ aUniversität Regensburg, Naturwissenschaftliche Fakultät I-Mathematik, \\ Univesitätsstraße 31, D-93053 Regensburg, Germany; 'Institute for Information \\ Transmission Problems, Russian Academy of Sciences, Bol. Karetny lane 19, \\ Moscow 101447, Russia
}

(Received 23 November 1999; In final form 26 January 2000)

The paper suggests sufficient conditions for the existence of positive solutions to vector boundary-value problems for second-order differential equations. For potential problems we develop the approach which combines the classical variational method with methods of positive operators. The results are then applied to nonpotential problems where the nonlinearities have potential minorants and majorants.

Keywords: Positive solution; Partial ordering generated by cone; Positive operator; Variational method

AMS Subject Classifications: Primary: 34B15; Secondary: 45M20

\section{INTRODUCTION}

The paper deals with the two-point problem for systems of secondorder differential equations. The problem is reduced in the standard way to an integral equation which can be written as

$$
x(t)=B \Gamma x(t) .
$$

*Tel.: +49941 9434918, Fax: +49941 9434005, e-mail: reinhard.mennicken@ mathematik.uni-regensburg.de

${ }^{\dagger}$ Corresponding author. Tel.: +7095 2998354, Fax: +7 095 2090579, e-mail: rach@iitp.ru 
Here $x(t), 0 \leq t \leq 1$, is a vector-valued function with the range $R^{N} ; \Gamma$ is the superposition operator $\Gamma: x(t) \rightarrow g(t, x(t))$ generated by the nonlinear continuous function $g(t, x) ; B$ is a linear integral operator with a continuous kernel. ${ }^{1}$ The operator $B$ is self-adjoint ${ }^{2}$ in $L^{2}$ and positive definite.

The nonlinearity $g(t, x)$ and the operator $\Gamma$ are said to be potential if $g(t, x)$ is the gradient (w.r.t. the variable $x$ ) of some scalar function $G(t, x)$, i.e., $g(t, x)=\nabla_{x} G(t, x)$. Equation (1) with the potential operator $\Gamma$ can be studied by variational methods (see, e.g. [1-3]). A general sufficient condition for the existence of a solution is that $G(t, x)$ satisfies the quadratic estimate ${ }^{3}$

$$
G(t, x) \leq \kappa|x|^{2}+b
$$

for all $x \in R^{N}, t \in[0,1]$ where $\kappa\|B\|_{L^{2} \rightarrow L^{2}}<1$ and $b$ is arbitrary.

In this paper, we are interested in positive solutions of Eq. (1) (the vector-valued function $x(t)$ is called positive if all its components are nonnegative functions). The problem considered leads to the equation with the positive definite self-adjoint operator $B$ that has additionally the following property:

For every sufficiently large $\alpha$ the operator $(I+\alpha B)^{-1} B$ is positive (in the sense of Krein [6]) w.r.t. semiordering generated by the cone of positive functions, i.e., this operator maps any positive function to a positive function.

This property allows to combine the variational method with methods of positive operators $[7,8]$ to obtain sufficient conditions for the existence of positive solutions to the boundary-value problem.

The paper is organized as follows. In Sections 2 and 3 problems with potential non-linearities $g(t, x)=\nabla_{x} G(t, x)$ are considered; it is supposed that $G(t, x)$ satisfies (2). Section 2 presents the main result on the existence of a positive solution. In Section 3 we suppose that $g(t, 0) \equiv 0$ (so the problem has the trivial zero solution) and suggest sufficient conditions for the existence of a nontrivial positive solution.

\footnotetext{
${ }^{1}$ The kernel of $B$ is the Green matrix-valued function of the two-point problem.

${ }^{2}$ Everywhere we use the spaces of vector-valued functions $x(t):[0,1] \rightarrow R^{N}$.

${ }^{3}$ This result was originally proved by Golomb [4] and Hammerstein [5] for integral equations.
} 
In Section 4, we consider applications to problems with nonpotential nonlinearities which have potential minorants and majorants. Sections 5 and 6 contain some remarks on the results obtained and the proofs.

The approach developed here or appropriate modifications can be used to prove the existence of positive solutions for problems on nonlinear oscillations, boundary-value problems for PDE etc.

\section{EXISTENCE OF POSITIVE SOLUTIONS}

Consider the problem

$$
-x^{\prime \prime}+A(t) x=g(t, x), \quad x(0)=x(1)=0
$$

where ${ }^{4} x \in R^{N}, N \geq 1$. Everywhere we suppose that the $N \times N$ real matrix $A(t)$ depends continuously on $t$ and its elements satisfy the relations

$$
a_{i j}(t)=a_{j i}(t) \leq 0 \quad \text { for all } i \neq j, i, j=1, \ldots, N ; t \in[0,1] .
$$

The function $g(t, x):[0,1] \times R^{N} \rightarrow R^{N}$ is continuous w.r.t. the set of its arguments.

Define the differential operator

$$
\begin{aligned}
& \mathcal{L} x(t)=-x^{\prime \prime}(t)+A(t) x(t) \text { with the boundary conditions } \\
& x(0)=x(1)=0
\end{aligned}
$$

now problem (3) can be written as $\mathcal{L} x(t)=g(t, x(t))$. Since the matrix $A(t)$ is symmetric, $\mathcal{L}$ is a symmetric operator in $L^{2}$. The spectrum $\sigma(\mathcal{L})$ of $\mathcal{L}$ (see e.g. [9]) is an increasing sequence of real eigenvalues

$$
\lambda_{0} \leq \lambda_{1} \leq \cdots \leq \lambda_{n} \leq \cdots, \quad \lambda_{n} \rightarrow \infty .
$$

In the following, we use the smallest eigenvalue $\lambda_{0}=\lambda_{0}(\mathcal{L})$.

\footnotetext{
${ }^{4}$ Throughout the paper the basis in $R^{N}$ is fixed; a vector $x \in R^{N}$ is identified with its coordinate column $\left(x_{1}, \ldots, x_{N}\right)^{T}$ or row $\left(x_{1}, \ldots, x_{N}\right)$. The notations $(\cdot, \cdot)$ and $|\cdot|$ are used for the usual scalar product $(x, y)=x_{1} y_{1}+\cdots+x_{N} y_{N}$ and the Eucledian norm $|x|=\sqrt{(x, x)}$.
} 
Suppose that $g(t, x)$ is a potential nonlinearity, i.e., its components are defined by

$$
g_{i}\left(t, x_{1}, \ldots, x_{N}\right)=\frac{\partial}{\partial x_{i}} G\left(t, x_{1}, \ldots, x_{N}\right)
$$

where $x=\left(x_{1}, \ldots, x_{N}\right)^{T} \in R^{N}, t \in[0,1]$. Without loss of generality, assume $^{5}$ that $G(t, 0) \equiv 0$. Therefore the function $G(t, x)$ is continuous w.r.t. the set of its arguments.

Denote by $K_{+}$the cone of the vectors $x \in R^{N}$ with nonnegative components:

$$
K_{+}=\left\{x=\left(x_{1}, \ldots, x_{N}\right)^{T} \in R^{N}: x_{i} \geq 0, i=1, \ldots, N\right\} .
$$

We write $x \geq y$ if $x-y \in K_{+}$and $x(t) \geqslant y(t)$ if $x(t) \geq y(t)$ for every $t \in[0,1]$. A function $x(t)$ is positive if $x(t) \geqslant 0$.

Theorem 1 Let (4) and (7) hold. Suppose that the function $G(t, x)$ satisfies estimate (2) with some $\kappa<\lambda_{0}(\mathcal{L}) / 2$ for any $x \in K_{+}$. Suppose

$$
x_{i}=0, x=\left(x_{1}, \ldots, x_{N}\right)^{T} \in K_{+} \quad \text { imply } \quad g_{i}(t, x) \geq 0
$$

for each $i=1, \ldots, N, t \in[0,1]$. Then problem (3) has a classical positive solution.

Consider problem (3) with the nonlinearity of the simple form

$$
g(t, x)=\left(g_{1}\left(t, x_{1}\right), \ldots, g_{N}\left(t, x_{N}\right)\right)
$$

where the component $g_{i}(\cdot, \cdot)$ depends on the component $x_{i}$ of the variable $x$ and $t$ only.

This is a potential nonlinearity, the function $G(t, x)$ is given by

$$
G(t, x)=\sum_{i=1}^{N} \int_{0}^{x_{i}} g_{i}(t, \xi) d \xi .
$$

Theorem 1 implies the following statement.

\footnotetext{
${ }^{5}$ If $G(t, 0)$ is not identically zero, it can be replaced by the function $\tilde{G}(t, x)=$ $G(t, x)-G(t, 0)$. Obviously, $\tilde{G}(t, 0) \equiv 0$ and $(7)$ implies that $g(t, x)$ is the gradient of $\tilde{G}(t, x)$ w.r.t. $x$.
} 
THEOREM 2 Suppose that relations (4) and (9) hold and the estimate

$$
\sum_{i=1}^{N} \int_{0}^{x_{i}} g_{i}(t, \xi) d \xi \leq \kappa \sum_{i=1}^{N} x_{i}^{2}+b, \quad x=\left(x_{1}, \ldots, x_{N}\right)^{T} \in K_{+}
$$

is valid with some $\kappa<\lambda_{0}(\mathcal{L}) / 2$. Suppose that $g(t, 0)$ is a positive function. Then problem (3) has a classical positive solution.

Nonlinearities of the form (9) are used below as minorants and majorants in problems with the nonpotential nonlinear part.

\section{NONZERO POSITIVE SOLUTIONS}

In this section it is supposed that $g(t, 0) \equiv 0$, so problem (3) has the zero solution.

Assume that (7) holds. To be simple, assume also that the function $g(t, x)$ is continuously differentiable in $x$, i.e., the Jacobian matrix

$$
J(t, x)=\left[\partial g_{i}(t, x) / \partial x_{j}\right]_{i, j=1, \ldots, N}
$$

exists for all $t \in[0,1], x \in R^{N}$ and depends continuously on its arguments. Relation (7) implies $J(t, x)=J^{T}(t, x)$.

Set $D(t)=J(t, 0)$ and consider the differential operator

$$
\begin{array}{r}
\mathcal{M} x(t)=-x^{\prime \prime}(t)+[A(t)-D(t)] x(t) \text { with the boundary conditions } \\
\qquad x(0)=x(1)=0 .
\end{array}
$$

Like (5), this is a symmetric operator ${ }^{6}$ and its spectrum is a sequence of eigenvalues $\mu_{0} \leq \mu_{1} \leq \cdots \mu_{n} \leq \cdots, \mu_{n} \rightarrow \infty$.

THEOREM 3 Let all the conditions of Theorem 1 be satisfied and $g(t, 0) \equiv 0$. Let the smallest eigenvalue $\mu_{0}=\mu_{0}(\mathcal{M})$ of operator (11) be negative. Then problem (3) has a classical nonzero positive solution $x^{*}(t)$.

Suppose all the conditions of Theorem 3 are fulfilled. If estimate (2) is valid for every $x \in K_{+} \cup\left\{-K_{+}\right\}$(not only for $x \in K_{+}$) and in

\footnotetext{
${ }^{6}$ The linearization of problem (3) at the origin leads to the problem $\mathcal{M} x(t)=0$.
} 
addition to (8) the relation

$$
x_{i}=0, x=\left(x_{1}, \ldots, x_{N}\right)^{T} \in\left\{-K_{+}\right\} \quad \text { imply } \quad g_{i}(t, x) \leq 0
$$

holds, then problem (3) has at least three solutions. Indeed, in this case the problem $\mathcal{L} y(t)=-g(t,-y(t))$, which is obtained from (3) by the change of the variable $y=-x$, also satisfies the conditions of Theorem 3, therefore it has a positive nonzero solution $y^{*}(t)$. Thus, besides the positive solution $x^{*}(t) \neq \equiv$ and the zero solution, problem (3) has the negative solution $-y^{*}(t) \not \equiv 0$.

If the nonlinearity $g(t, x)$ has the form (9), then the matrix $D(t)$ is diagonal, i.e., $D(t)=\operatorname{diag}\left\{d_{11}(t), \ldots, d_{N N}(t)\right\}$ where $d_{i i}(t)=\partial g_{i}\left(t, x_{i}\right) /$ $\partial x_{i}$.

Theorem 4 Let relations (4), (9), and $g(t, 0) \equiv 0$ be valid. Suppose $\mu_{0}<0$ and estimate (10) holds for some $\kappa<\lambda_{0} / 2$ where $\lambda_{0}, \mu_{0}$ are the smallest eigenvalues of operators (5) and (11). Then problem (3) has a classical nonzero positive solution.

Theorem 4 follows from Theorem 3 .

\section{NONPOTENTIAL PROBLEMS}

In this section the nonlinearity in (3) is not supposed to be potential. To stress this we use the new notation for the nonlinearity, so we consider the problem

$$
-x^{\prime \prime}+A(t) x=f(t, x), \quad x(0)=x(1)=0
$$

where $A(t)$ satisfies (4). The main assumption about the continuous function $f(t, x)$ is that the estimates

$$
h_{i}\left(t, x_{i}\right) \leq f_{i}\left(t, x_{1}, \ldots, x_{N}\right) \leq g_{i}\left(t, x_{i}\right), \quad i=1, \ldots, N
$$

are valid on some domains specified below. Here $h_{i}(t, \xi), g_{i}(t, \xi)$ are smooth functions of their arguments $t \in[0,1], \xi \in R$.

Define the vector-valued functions

$$
\begin{aligned}
& h(t, x)=\left(h_{1}\left(t, x_{1}\right), \ldots, h_{N}\left(t, x_{N}\right)\right), \\
& g(t, x)=\left(g_{1}\left(t, x_{1}\right), \ldots, g_{N}\left(t, x_{N}\right)\right)
\end{aligned}
$$


and consider the auxiliary problems

$$
-x^{\prime \prime}+A(t) x=h(t, x), \quad-x^{\prime \prime}+A(t) x=g(t, x), \quad x(0)=x(1)=0 .
$$

Lemma 1 Suppose that problems (15) have solutions $x_{-}(t), x_{+}(t)$ satisfying $x_{-}(t) \leqslant x_{+}(t)$. Let estimates (13) be valid for every pair $\{t, x\} \in[0,1] \times R^{N}$ such that $x_{-}(t) \leq x \leq x_{+}(t)$. Then problem (12) has a solution $x^{*}(t)$ satisfying

$$
x_{-}(t) \leq x^{*}(t) \leq x_{+}(t) .
$$

We combine Lemma 1 with Theorems 2 and 4 to obtain sufficient conditions for the existence of positive solutions to the nonpotential problem (12).

Let

$$
A(t)=\operatorname{diag}\left\{A_{1}(t), \ldots, A_{k}(t)\right\}
$$

here $A_{i}(t)$ is a symmetric square matrix of order $N_{i}-N_{i-1}$ where ${ }^{7}$ $0=N_{0}<N_{1}<\cdots<N_{k}=N, 1 \leq k \leq N$. Denoteby $\lambda_{0 i}$ the smallest eigenvalue of the differential operator $\mathcal{L}_{i} z(t)=-z^{\prime \prime}(t)+A_{i}(t) z(t)$ with the boundary conditions $z(0)=z(1)=0$ which acts in the space of vector functions $z(t):[0,1] \rightarrow R^{N_{i}-N_{i-1}}$.

THeOREM 5 Let (4) and (17) be satisfied. Let

$$
x_{j}=0, x=\left(x_{1}, \ldots, x_{N}\right)^{T} \in K_{+} \quad \text { imply } f_{j}(t, x) \geq 0
$$

and

$$
f_{j}\left(t, x_{1}, \ldots, x_{N}\right) \leq g_{j}\left(t, x_{j}\right) \quad \text { whenever } \quad x=\left(x_{1}, \ldots, x_{N}\right)^{T} \in K_{+}
$$

for every $1 \leq j \leq N$. Suppose that for each $i=1, \ldots, k$ there are a $\kappa_{i}<\lambda_{0 i} / 2$ and $a b_{i}>0$ such that the estimate

$$
\begin{gathered}
\sum_{j=N_{i-1}+1}^{N_{i}} \int_{0}^{x_{j}} g_{j}(t, \xi) d \xi \leq \kappa_{i} \sum_{j=N_{i-1}+1}^{N_{i}} x_{j}^{2}+b_{i} \\
\text { if } x_{N_{i-1}+1} \geq 0, \ldots, x_{N_{i}} \geq 0
\end{gathered}
$$

holds. Then problem (12) has a classical positive solution.

\footnotetext{
${ }^{7}$ Evidently, each symmetric $N \times N$ matrix $A(t)$ has the form (17) with $k=1$.
} 
The minorant $h(t, x)$ of the nonlinearity $f(t, x)$ is not mentioned in the formulation of Theorem 5 , it is constructed in the proof.

Now consider problem (12) where $f(t, 0) \equiv 0$, i.e., the problem with the zero solution. Suppose that $f(t, x)$ satisfies two-sided estimates (13) for all $x \in K_{+}$and set

$$
d_{j}(t)=\frac{\partial h_{j}}{\partial \xi}(t, 0), \quad D_{i}(t)=\operatorname{diag}\left\{d_{N_{i-1}+1}(t), \ldots, d_{N_{i}}(t)\right\} .
$$

Denote by $\mu_{0 i}$ the smallest eigenvalue of the differential operator $\mathcal{M}_{i} z(t)=-z^{\prime \prime}(t)+\left[A_{i}(t)-D_{i}(t)\right] z(t)$ with the boundary conditions $z(0)=z(1)=0$.

Theorem 6 Let (4) and (17) be satisfied. Suppose that estimates (13) are valid for all $x \in K_{+}$and $h(t, 0) \equiv f(t, 0) \equiv 0$. Suppose estimate (19) with $\kappa_{i}<\lambda_{0 i} / 2$ holds for each $i=1, \ldots, k$ and

$$
\min \left\{\mu_{01}, \ldots, \mu_{0 k}\right\}<0 .
$$

Then problem (12) has a classical nonzero positive solution.

\section{REMARKS}

(a) In the theorems above on the existence of positive solutions to problem (3) it is supposed that the nonlinearity $g(t, x)$ is defined for all $x$. Naturally, one can consider the nonlinearities defined for $x \in K_{+}$only. For example, Theorem 1 is true without any change in formulation if the continuous function (7) is defined for $x \in K_{+}$, $t \in[0,1]$ (it is necessary just to remember that $g_{i}(t, x)$ is now the one-sided derivative of $G(t, x)$ whenever $\left.x_{i}=0\right)$. This follows from the proof presented in the next section.

This remark is applicable to Theorem 3 as well.

(b) We say that the function $f(t, x):[0,1] \times R^{N} \rightarrow R^{N}$ increases in the off-diagonal variables if for every $i=1, \ldots, N$ the relations $\left(x_{1}, \ldots, x_{N}\right)^{T}=x \geq y=\left(y_{1}, \ldots, y_{N}\right)^{T}, x_{i}=y_{i}$ imply the estimate $f_{i}(t, x) \geq f_{i}(t, y), 0 \leq t \leq 1$. Suppose that the estimates

$$
h(t, x) \leq f(t, x) \leq g(t, x)
$$


with smooth $h(\cdot, \cdot), g(\cdot, \cdot)$ are valid for $t \in[0,1], x \in R^{N}$ and problems (15) have the solutions $x_{-}(t), x_{+}(t)$ satisfying

$$
x_{-}(t) \leq x_{+}(t)
$$

like in Lemma 1. We do not assume here that the functions $h(t, x)$ and $g(t, x)$ have the form (14). Nevertheless, if either the function $f(t, x)$ or both the functions $h(t, x), g(t, x)$ increase in the offdiagonal variables, then the conclusion of Lemma 1 is true, i.e., problem (12) has a solution satisfying (16). In this case, one can use Theorems 1, 3 to obtain sufficient conditions for the existence of positive solutions to problem (12) in the same way as Theorems 5, 6 are derived from Theorems 2, 4 and Lemma 1 (see the proofs below).

(c) Consider the problem

$$
-x^{\prime \prime}+A(t) x=f\left(t, x, x^{\prime}\right), \quad x(0)=x(1)=0
$$

where $A(t)$ satisfies (4) and $f(t, x, y):[0,1] \times R^{N} \times R^{N} \rightarrow R^{N}$ is a continuous function. For this problem analogs of Lemma 1 are also valid. In particular, if

$$
x_{-}(t) \leq x \leq x_{+}(t) \quad \text { imply } h_{i}\left(t, x_{i}\right) \leq f_{i}(t, x, y) \leq g_{i}\left(t, x_{i}\right)
$$

for every $t \in[0,1], y \in R^{N}, i=1, \ldots, N$ where $x_{-}(t), x_{+}(t)$ are the solutions (21) of problems (15), then problem (22) has a solution satisfying (16) (see, also [10]). This leads to analogs of Theorems 5 and 6 for problem (22).

\section{PROOF OF THEOREMS 1 AND 3}

\subsection{The Linear Operator}

First we recall some well-known facts about the operator $\mathcal{L}$. Consider the linear problem

$$
\mathcal{L} x(t)+\lambda x(t)=u(t) .
$$

Suppose $-\lambda$ is not an eigenvalue of the operator $\mathcal{L}$, i.e., $\lambda \neq-\lambda_{n}$, $n=0,1,2, \ldots$. Then a unique solution of problem (23) is given by the 
formula (see, e.g. [9])

$$
x(t)=B_{\lambda} u(t):=\int_{0}^{1} H_{\lambda}(t, s) u(s) d s
$$

where the Green matrix-valued function $H_{\lambda}(t, s)$ is continuous w.r.t. the set of its arguments $0 \leq s, t \leq 1$ and satisfies

$$
H_{\lambda}(t, s)=H_{\lambda}^{T}(s, t), \quad 0 \leq s, t \leq 1 .
$$

If $u(t) \in C$ then $x(t)=B_{\lambda} u(t)$ is a classical solution of problem (23). By continuity of $H_{\lambda}(t, s)$, the solution $x(t)=B_{\lambda} u(t)$ is continuous for every $u(t) \in L^{1}$ and moreover, the operator $B_{\lambda}$ is completely continuous from $L^{1}$ to $C$ (also, it is continuous from $C$ to $C^{2}$ ).

Relation (24) implies that $B_{\lambda}=B_{\lambda}^{*}$, i.e., $B_{\lambda}$ is a self-adjoint operator in $L^{2}$. Since $B_{\lambda}$ is the inverse of the operator $\mathcal{L}+\lambda I$, the spectrum of $B_{\lambda}$ is the sequence

$$
\beta_{n}(\lambda)=\left(\lambda+\lambda_{n}\right)^{-1}, \quad n=0,1,2, \ldots
$$

If $\lambda>-\lambda_{0}$ then $\left(\lambda+\lambda_{0}\right)^{-1}=\beta_{0}(\lambda) \geq \beta_{1}(\lambda) \geq \ldots \geq \beta_{n}(\lambda) \geq \ldots>0$, hence the operator $B_{\lambda}$ is positive definite and

$$
0 \leq\left(w, B_{\lambda} w\right)_{L^{2}} \leq\left(\lambda+\lambda_{0}\right)^{-1}\|w\|_{L^{2}}^{2}, \quad w \in L^{2}
$$

where $(\cdot, \cdot)_{L^{2}}$ denotes the scalar product in $L^{2}$. Therefore, for each $\lambda>-\lambda_{0}$ the self-adjoint positive definite square $\operatorname{root} B_{\lambda}^{(1 / 2)}$ of the operator $B_{\lambda}$ is defined. ${ }^{8}$ By splitting theorems (see, e.g. [11]), $B_{\lambda}^{(1 / 2)}$ is a completely continuous operator from $L^{1}$ to $L^{2}$ and from $L^{2}$ to $C$.

Below we use the operators $B_{\lambda}$ and $B_{\lambda}^{(1 / 2)}$ with various $\lambda>-\lambda_{0}$. The operators $B_{\lambda}$ satisfy the resolvent equation

$$
B_{\mu}-B_{\lambda}=(\lambda-\mu) B_{\mu} B_{\lambda}
$$

for every $\lambda, \mu \neq-\lambda_{n}, n=0,1,2, \ldots$.

\subsection{Equivalent Problem and Operator Equation}

Define

$$
P x=\frac{1}{2}\left(x_{1}+\left|x_{1}\right|, \ldots, x_{N}+\left|x_{N}\right|\right)^{T} .
$$

\footnotetext{
${ }^{8}$ The square root $B_{\lambda}^{(1 / 2)}$ of $B_{\lambda}$ is not necessarily an integral operator.
} 
The operator $P$ projects the space $R^{N}$ onto the cone $K_{+}$and satisfies the estimates $|P x| \leq|x|$ and $|P x-P y| \leq|x-y|$ for all $x, y \in R^{N}$. Take an $\alpha \geq 0$ and set

$$
\bar{g}_{i}(t, x ; \alpha)=\left\{\begin{array}{cc}
g_{i}(t, P x) & \text { for } x_{i} \geq 0 \\
-2 \alpha x_{i} & \text { for } x_{i}<0
\end{array}\right.
$$

for every $x=\left(x_{1}, \ldots, x_{N}\right)^{T} \in R^{N}, i=1, \ldots, N$. We replace problem (3) by the problem

$$
\mathcal{L} x(t)=\bar{g}(t, x(t) ; \alpha)
$$

here the components of the function $\bar{g}(t, x ; \alpha):[0,1] \times R^{N} \rightarrow R^{N}$ are defined by (27); $\alpha$ is a parameter. Since $\bar{g}(t, x ; \alpha)=g(t, x)$ for $x \in K_{+}$, the classical positive solutions of problems (3) and (28) coincide for every $\alpha$.

Note that the function $\bar{g}(t, x ; \alpha)$ with a fixed $t$ is discontinuous in $x$, its discontinuity points lie in the hyperplanes $x_{i}=0$. The set of all discontinuity points is

$$
\Pi_{t}=\bigcup_{i=1}^{N}\left\{x=\left(x_{1}, \ldots, x_{N}\right)^{T}: x_{i}=0, g_{i}(t, P x) \neq 0\right\} .
$$

Define

$$
\bar{G}(t, x ; \alpha)=G(t, P x)-\alpha|x-P x|^{2}, \quad x \in R^{N} .
$$

From (7) and (27) it follows that

$$
\bar{g}_{i}(t, x ; \alpha)=\frac{\partial}{\partial x_{i}} \bar{G}(t, x ; \alpha), \quad i=1, \ldots, N
$$

for every $x \in R^{N} \backslash \Pi_{t}, t \in[0,1]$. It is readily seen that

$$
\bar{G}(t, x+y ; \alpha)-\bar{G}(t, x ; \alpha)=\int_{0}^{1}(\bar{g}(t, x+s y ; \alpha), y) d s, \quad x, y \in R^{N} .
$$

Let us rewrite (28) in the form

$$
\mathcal{L} x(t)+\lambda x(t)=\bar{g}(t, x(t) ; \alpha)+\lambda x(t), \quad \lambda>-\lambda_{0}
$$


and pass from problem (32) to the equivalent integral equation

$$
x(t)=B_{\lambda}[\bar{g}(t, x(t) ; \alpha)+\lambda x(t)]
$$

with the two parameters $\alpha \geq 0$ and $\lambda>-\lambda_{0}$. We consider Eq. (33) in the space $C$ : by the inclusion $x(t) \in C$ implies $g(t, x(t) ; \alpha) \in L^{\infty}$ and since $B_{\lambda}$ is a completely continuous operator from $L^{\infty}$ to $C$, it follows that the operator $B_{\lambda}[\bar{g}(t, x(t) ; \alpha)+\lambda x(t)]$ on the right-hand side of (33) is completely continuous in $C$. If $x(t) \geqslant 0$ then the function

$$
\bar{g}(t, x(t) ; \alpha)+\lambda x(t)=g(t, x(t))+\lambda x(t)
$$

is continuous, so the operator $B_{\lambda}$ maps it to $C^{2}$. Therefore every positive solution of Eq. (33) is a classical positive solution of problems (32) and (3). It is important to note that by construction, Eq. (33) with a fixed $\alpha$ are equivalent ${ }^{9}$ for all $\lambda>-\lambda_{0}$.

Now, to prove Theorem 1 it suffices to show that for some pair $\alpha, \lambda$ Eq. (33) has a solution $x^{*}(t) \in C, x^{*}(t) \geqslant 0$. In Theorem 3 it is necessary to show in addition that $x^{*}(t)$ is not the zero solution.

\subsection{Main Steps of the Proof}

We prove the existence of a positive solution to Eq. (33) in two steps, using the two lemmas below; the proofs of the lemmas are given in the next subsections. First, it is shown by the variational method that for every $\alpha>0$ Eq. (33) has a solution $x_{\alpha}(t)$ satisfying the uniform estimate $\left\|x_{\alpha}(t)\right\|_{C} \leq R_{0}$ with the $R_{0}$ independent of $\alpha$. In the second step, we prove that the solution $x_{\alpha}(t)$ is positive for every sufficiently large $\alpha$.

Take any $\alpha \geq 0$ and fix it up to the end of the first step. Set $\nu=-2 \kappa$; by assumption, $\nu>-\lambda_{0}$. Following the general variational scheme (see, e.g. $[1,2]$ ), consider the functional

$$
V_{\alpha}(w)=\frac{1}{2}(w, w)_{L^{2}}-\frac{\nu}{2}\left(w, B_{\nu} w\right)_{L^{2}}-\int_{0}^{1} \bar{G}\left(t, B_{\nu}^{(1 / 2)} w(t) ; \alpha\right) d t, \quad w \in L^{2} .
$$

\footnotetext{
${ }^{9}$ Also, one can see this from resolvent Eq. (26): applying the operator $I+(\lambda-\mu) B_{\mu}$ to equality (33) one obtains $x(t)+(\lambda-\mu) B_{\mu} x(t)=\left(B_{\lambda}+(\lambda-\mu) B_{\mu} B_{\lambda}\right)(\bar{g}(t, x(t) ; \alpha)+\lambda x(t))$ and (26) gives $x(t)=B_{\mu}(\bar{g}(t, x(t) ; \alpha)+\mu x(t))$.
} 
Since $w(t) \in L^{2}$ implies $B_{\nu}^{(1 / 2)} w(t) \in C$ and the function $\bar{G}(t, x ; \alpha)$ is continuous w.r.t. the set of its arguments $t, x$, the functional $V_{\alpha}(w)$ is defined on the whole space $L^{2}$.

Denote by $\mathcal{E}$ the set of the functions $w \in L^{2}$ satisfying

$$
\operatorname{mes}\left\{t \in[0,1]: B_{\nu}^{(1 / 2)} w(t) \in \Pi_{t}\right\}=0
$$

where $\Pi_{t}$ is set (29). We claim that the functional $V_{\alpha}(w)$ is Frechet differentiable at every point $w \in \mathcal{E}$ and its derivative is given by

$$
\nabla V_{\alpha}(w)=w(t)-\nu B_{\nu} w(t)-B_{\nu}^{(1 / 2)} \bar{g}\left(t, B_{\nu}^{(1 / 2)} w(t) ; \alpha\right), \quad w=w(t) \in \mathcal{E} .
$$

If $w_{*} \in \mathcal{E}$ is a critical point of the functional $V_{\alpha}(w)$, then (35) implies

$$
w_{*}(t)=B_{\nu}^{(1 / 2)}\left(\bar{g}\left(t, B_{\nu}^{(1 / 2)} w_{*}(t) ; \alpha\right)+\nu B_{\nu}^{(1 / 2)} w_{*}(t)\right),
$$

therefore each critical point $w_{*} \in \mathcal{E}$ determines a continuous solution $x_{*}(t)=B_{\nu}^{(1 / 2)} w_{*}(t)$ of Eq. (33) with $\lambda=\nu$ and hence with any $\lambda>-\lambda_{0}$.

Evidently, $w-\nu B_{\nu} w$ is the Frechet derivative of the functional $1 / 2\left((w, w)_{L^{2}}-\nu\left(w, B_{\nu} w\right)_{L^{2}}\right)$. So to prove relation (35), we need to show that the functional

$$
\mathcal{F}_{\alpha}(w):=\int_{0}^{1} \bar{G}\left(t, B_{\nu}^{(1 / 2)} w(t) ; \alpha\right) d t, \quad w \in L^{2}
$$

satisfies

$$
\lim _{n \rightarrow \infty}\left\|h_{n}\right\|_{L^{2}}^{-1}\left(\mathcal{F}_{\alpha}\left(w+h_{n}\right)-\mathcal{F}_{\alpha}(w)-\left(h_{n}, B_{\nu}^{(1 / 2)} \bar{g}\left(\cdot, B_{\nu}^{(1 / 2)} w(\cdot) ; \alpha\right)\right)_{L^{2}}\right)=0
$$

for every $w \in \mathcal{E}$ and every vanishing sequence $h_{n} \in L^{2}$. Substituting $x(t)=B_{\nu}^{(1 / 2)} w(t), \quad y=B_{\nu}^{(1 / 2)} h(t)$ in (31) and integrating over the segment $[0,1]$, we obtain

$$
\begin{array}{r}
\mathcal{F}_{\alpha}(w+h)-\mathcal{F}_{\alpha}(w)=\int_{0}^{1} \int_{0}^{1}\left(B_{\nu}^{(1 / 2)} h(t), \bar{g}\left(t, B_{\nu}^{(1 / 2)} w(t)\right.\right. \\
\left.\left.+s B_{\nu}^{(1 / 2)} h(t) ; \alpha\right)\right) d s d t
\end{array}
$$


Also note that $B_{\nu}^{(1 / 2)}=\left(B_{\nu}^{(1 / 2)}\right)^{*}$ implies

$$
\left(h_{n}, B_{\nu}^{(1 / 2)} \bar{g}\left(\cdot,\left(B_{\nu}^{(1 / 2)} w(\cdot) ; \alpha\right)\right)_{L^{2}}=\int_{0}^{1}\left(B_{\nu}^{(1 / 2)} h_{n}(t), \bar{g}\left(t, B_{\nu}^{(1 / 2)} w(t) ; \alpha\right)\right) d t\right.
$$

therefore (38) is equivalent to

$$
\begin{gathered}
\lim _{n \rightarrow \infty}\left\|h_{n}\right\|_{L^{2}}^{-1} \int_{0}^{1} \int_{0}^{1}\left(B_{\nu}^{(1 / 2)} h_{n}(t), \bar{g}\left(t, B_{\nu}^{(1 / 2)} w(t)+s B_{\nu}^{(1 / 2)} h_{n}(t) ; \alpha\right)\right. \\
\left.-\bar{g}\left(t, B_{\nu}^{(1 / 2)} w(t) ; \alpha\right)\right) d s d t=0
\end{gathered}
$$

The inclusion $w \in \mathcal{E}$ means that for a.e. $t \in[0,1]$ the point $x_{0}=x_{0}(t)=$ $B_{\nu}^{(1 / 2)} w(t)$ is a continuity point of the function $\bar{g}(t, x ; \alpha)$ (considered as a function of $x$ with the fixed $t$ ). From $\left\|h_{n}\right\|_{L^{2}} \rightarrow 0$ it follows $\left\|B_{\nu}^{(1 / 2)} h_{n}\right\|_{C} \rightarrow 0$, hence

$$
\lim _{n \rightarrow \infty}\left|\bar{g}\left(t, x_{0}(t)+s B_{\nu}^{\frac{1}{2}} h_{n}(t) ; \alpha\right)-\bar{g}\left(t, x_{0}(t) ; \alpha\right)\right|=0 \quad \text { for a.e. } t \in[0,1]
$$

and for each $s \in[0,1]$. But the function $\bar{g}(t, x ; \alpha)$ is locally bounded and we have $\sup \left\{\left\|x_{0}(t)+s B_{\nu}^{(1 / 2)} h_{n}(t)\right\|_{C}: s \in[0,1], n=1,2, \ldots\right\}<\infty$, hence

$$
\sup _{n} \sup _{0 \leq t, s \leq 1}\left|\bar{g}\left(t, x_{0}(t)+s B_{\nu}^{\frac{1}{2}} h_{n}(t) ; \alpha\right)-\bar{g}\left(t, x_{0}(t) ; \alpha\right)\right|<\infty,
$$

and by the Lesbegue theorem, (41) implies

$$
\lim _{n \rightarrow \infty} \int_{0}^{1} \int_{0}^{1}\left|\bar{g}\left(t, B_{\nu}^{\frac{1}{2}} w(t)+s B_{\nu}^{\frac{1}{2}} h_{n}(t) ; \alpha\right)-\bar{g}\left(t, B_{\nu}^{\frac{1}{2}} w(t) ; \alpha\right)\right| d s d t=0 .
$$

Since $\left\|B_{\nu}^{(1 / 2)} h_{n}\right\|_{C}\left\|h_{n}\right\|_{L^{2}}^{-1} \leq\left\|B_{\nu}^{(1 / 2)}\right\|_{L^{2} \rightarrow C}$, this yields (40), thus (35) is proved.

The following lemma guarantees the existence of a critical point $w^{*} \in \mathcal{E}$ for the functional $V_{\alpha}(w)$.

Lemma 2 Let the assumptions of Theorem 1 be satisfied. Then the functional $V_{\alpha}(w)$ has a point $w_{\alpha}^{*}$ of global minimum. The function $w_{\alpha}^{*}=$ $w_{\alpha}^{*}(t)$ satisfies $w_{\alpha}^{*} \in \mathcal{E}$ and

$$
\left\|w_{\alpha}^{*}\right\|_{L^{2}} \leq \sqrt{2 b}
$$

where $b$ comes from (2). If in addition all the assumptions of Theorem 3 hold, then the function $w_{\alpha}^{*}(t)$ is not the zero solution. 
The lemma is proved in Subsection 6.5.

By Lemma $2, \nabla V_{\alpha}\left(w_{\alpha}^{*}\right)=0, w_{\alpha}^{*} \in \mathcal{E}$, so the function $x_{\alpha}(t)=$ $B_{\nu}^{(1 / 2)} w_{\alpha}^{*}(t)$ is a solution of Eq. (33) for every $\lambda>-\lambda_{0}$. From (42) the estimate $\left\|x_{\alpha}\right\|_{C} \leq R_{0}$ follows with

$$
R_{0}=\left\|B_{\nu}^{(1 / 2)}\right\|_{L^{2} \rightarrow C} \sqrt{2 b}
$$

which is independent of $\alpha$. To complete the first step of the proof note that the relation $\nabla V_{\alpha}\left(w_{\alpha}^{*}\right)=0$ can be written as

$$
w_{\alpha}^{*}(t)=B_{\nu}^{(1 / 2)}\left(\bar{g}\left(t, x_{\alpha}(t) ; \alpha\right)+\nu x_{\alpha}(t)\right) .
$$

If all the assumptions of Theorem 3 are valid, then $\bar{g}(t, 0 ; \alpha) \equiv g(t, 0) \equiv 0$, also by Lemma $2, w_{\alpha}^{*} \neq 0$. Thus, (44) implies that $x_{\alpha}(t) \not \equiv 0$ under the assumptions of Theorem 3.

The second step of the proof of Theorems 1 and 3 is based on the following fact.

Lemma 3 Relation (4) implies that for every sufficiently large $\lambda$ the operator $B_{\lambda}$ is positive ${ }^{10}$ w.r.t. to the cone of positive functions in $C$, i.e., $x(t) \geqslant 0$ implies $B_{\lambda} x(t) \geqslant 0$.

Lemma 3 is proved in the next subsection.

Now, substitute the function $x_{\alpha}(t)$ in (33) with $\lambda=\alpha$ to obtain the identity

$$
x_{\alpha}(t)=B_{\alpha}\left[\bar{g}\left(t, x_{\alpha}(t) ; \alpha\right)+\alpha x_{\alpha}(t)\right] .
$$

Suppose that either the function $g(t, x)$ has continuous derivatives $\partial g_{i}(t, x) / \partial x_{i}, i=1, \ldots, N$ and satisfies (8) (like in Theorem 3 ) or the relations

$$
x_{i}=0, x=\left(x_{1}, \ldots, x_{N}\right)^{T} \in K_{+} \quad \text { imply } \quad g_{i}(t, x) \geq \varepsilon
$$

are valid for all $i=1, \ldots, N$ with some $\varepsilon>0$. It follows easily from the definition of the function $\bar{g}(t, x ; \alpha)$ that in both cases the estimate

$$
\bar{g}(t, x ; \alpha)+\alpha x \geq 0, \quad t \in[0,1],|x| \leq R_{0}
$$

holds for every sufficiently large $\alpha$. Since $\left\|x_{\alpha}\right\|_{C} \leq R_{0}$, the function $\bar{g}\left(t, x_{\alpha}(t) ; \alpha\right)+\alpha x_{\alpha}(t)$ (with $\alpha$ large enough) is positive, and by Lemma

\footnotetext{
${ }^{10}$ One can show that the operator $B_{\lambda}$ is positive for every $\lambda>-\lambda_{0}$, but we do not use this fact here.
} 
3 the operator $B_{\alpha}$ maps it to the positive function (45). Thus, $x_{\alpha}(t)$ is a positive solution of Eq. (33) for every large $\alpha$.

This completes the proof of Theorem 3. This also proves the conclusion of Theorem 1 if in its assumptions estimate (8) is replaced by the stronger estimate (46).

We complete the proof of Theorem 1 with the following limit construction. Set $\tilde{g}_{i}(t, x ; \varepsilon):=g_{i}(t, x)+\varepsilon, i=1, \ldots, N$ and consider the problem

$$
\mathcal{L} x(t)=\tilde{g}(t, x(t) ; \varepsilon), \quad \varepsilon>0 .
$$

From (8) it follows that

$$
x_{i}=0, x=\left(x_{1}, \ldots, x_{N}\right)^{T} \in K_{+} \quad \text { imply } \quad \tilde{g}_{i}(t, x ; \varepsilon) \geq \varepsilon
$$

for all $i=1, \ldots, N$, similar to (46). Furthermore, relations (7) and (2) with $\kappa<\lambda_{0} / 2$ imply the relations

$$
\tilde{g}_{i}(t, x ; \varepsilon)=\frac{\partial}{\partial x_{i}}\left(G(t, x)+\varepsilon\left(x_{1}+x_{2}+\cdots+x_{N}\right)\right), \quad i=1, \ldots, N
$$

and

$$
G(t, x)+\varepsilon\left(x_{1}+x_{2}+\cdots+x_{N}\right) \leq\left(\kappa+\varepsilon_{0}\right)|x|^{2}+\left(b+N \varepsilon_{0}\right)
$$

for all $0<\varepsilon \leq \varepsilon_{0}$ where we take $\varepsilon_{0}>0$ sufficiently small so that $\kappa+\varepsilon_{0}<\lambda_{0} / 2$. Therefore problem (47) with $\varepsilon \in\left(0, \varepsilon_{0}\right)$ satisfies all the assumptions of Theorem 1 and estimate (48) guarantees that problem (47) has a classical positive solution $x_{\varepsilon}(t)$ satisfying $\left\|x_{\varepsilon}(t)\right\| \leq R_{\varepsilon_{0}}$, where $R_{\varepsilon_{0}}$ is defined by formula (43) with $b^{\prime}=b+N \varepsilon_{0}$ and $\nu^{\prime}=$ $-2\left(\kappa+\varepsilon_{0}\right)$ in place of $b$ and $\nu$. Replacing problem (47) by the equivalent integral equation $x(t)=B_{\lambda}(\tilde{g}(t, x(t) ; \varepsilon)+\lambda x(t))$, we obtain

$$
x_{\varepsilon}(t)=B_{\lambda}\left(\tilde{g}\left(t, x_{\varepsilon}(t) ; \varepsilon\right)+\lambda x_{\varepsilon}(t)\right), \quad 0<\varepsilon \leq \varepsilon_{0} .
$$

But $B_{\lambda}: C \rightarrow C$ is a completely continuous operator, therefore the estimates

$$
\left\|x_{\varepsilon}\right\| \leq R_{\varepsilon_{0}}, \quad \sup \left\{|\tilde{g}(t, x ; \varepsilon)|: t \in[0,1], 0<\varepsilon \leq \varepsilon_{0},|x| \leq R_{\varepsilon_{0}}\right\}<\infty
$$

imply that the set of functions (50) is compact in $C$, hence this set has a limit point $x^{*}(t) \in C$. Finally, passing to the limit in (50) as $\varepsilon \rightarrow+0$, we 
see that $x^{*}(t)$ satisfies the equation $x(t)=B_{\lambda}(g(t, x(t))+\lambda x(t))$, i.e., $x^{*}(t)$ is a classical solution of problem (3). Since $x_{\varepsilon}(t) \geqslant 0,0<\varepsilon \leq \varepsilon_{0}$, it follows that $x^{*}(t) \geqslant 0$ and Theorem 1 is proved.

\subsection{Proof of Lemma 3}

Denote by $\mathcal{Q}_{\mu}$ the operator that maps a function $v(t)$ to a unique solution $x(t)=\mathcal{Q}_{\mu} v(t)$ of the vector problem

$$
-x^{\prime \prime}+\mu x=v(t), \quad x(0)=x(1)=0
$$

which is the system of the independent scalar problems $-x_{i}^{\prime \prime}+\mu x_{i}=$ $v_{i}(t), x_{i}(0)=x_{i}(1)=0$. The integral operator $\mathcal{Q}_{\mu}$ can be written explicitely, it is well-known that $\mathcal{Q}_{\mu}$ is positive, i.e., $v(t) \geqslant 0$ implies $\mathcal{Q}_{\mu} v(t) \geq 0$, for every $\mu>-\pi^{-2}$. The norm of $\mathcal{Q}_{\mu}$ in $L^{2}$ is

$$
\left\|\mathcal{Q}_{\mu}\right\|_{L^{2} \rightarrow L^{2}}=\left(\mu+\pi^{-2}\right)^{-1}, \quad \mu>-\pi^{-2} .
$$

Set $\gamma:=\max \left\{\left\|a_{i i}(t)\right\|_{C}: i=1, \ldots, N\right\}$ where $a_{i i}(t)$ is the diagonal element of the matrix $A(t)$. Take any $u(t) \in C$ and put $\hat{x}(t)=B_{\mu-\gamma} u(t)$ where $\mu$ is sufficiently large. By definition, $\hat{x}(t)$ is the classical solution of the problem

$$
-x^{\prime \prime}(t)+A(t) x(t)+(\mu-\gamma) x(t)=u(t), \quad x(0)=x(1)=0,
$$

therefore $\hat{x}(t)$ is also the classical solution of problem (51) with $v(t)=u(t)+\gamma x(t)-A(t) x(t)$, hence $\hat{x}(t)=\mathcal{Q}_{\mu}(u(t)+\gamma \hat{x}(t)-A(t) \hat{x}(t))$. Equivalently,

$$
B_{\mu-\gamma} u(t)-\mathcal{Q}_{\mu}\left([\gamma E-A(t)] B_{\mu-\gamma} u(t)\right)=\mathcal{Q}_{\mu} u(t), \quad u(t) \in C,
$$

where $E$ is the $N \times N$ identity matrix. Denote by $\varphi=\varphi(t)$ the norm of the matrix $\gamma E-A(t)$ and by $\Phi$ the operator $\Phi: v(t) \rightarrow[\gamma E-A(t)]$ $v(t)$. From (52) the estimate $\left\|\mathcal{Q}_{\mu} \Phi\right\|_{L^{2} \rightarrow L^{2}} \leq\|\varphi(t)\|_{C}\left(\mu+\pi^{-2}\right)^{-1}$ follows. Therefore, for every sufficiently large $\mu$ the operator $I-$ $\mathcal{Q}_{\mu} \Phi$ is invertible in $L^{2}$ and the inverse is represented by Neumann series

$$
\left(I-\mathcal{Q}_{\mu} \Phi\right)^{-1}=I+\mathcal{Q}_{\mu} \Phi+\cdots+\left(\mathcal{Q}_{\mu} \Phi\right)^{n}+\cdots
$$


so applying this operator to (53), one obtains

$$
B_{\mu-\gamma} u(t)=u(t)+\mathcal{Q}_{\mu} \Phi \mathcal{Q}_{\mu} u(t)+\cdots+\mathcal{Q}_{\mu}\left(\Phi \mathcal{Q}_{\mu}\right)^{n} u(t)+\cdots, \quad u(t) \in C
$$

where the series converges ${ }^{11}$ in $L^{2}$. Finally, relations (4) and the estimate $\gamma \geq a_{i i}(t)$ imply that all the elements of the matrix $\gamma E-A(t)$ are nonnegative for each $t$, hence the operator $\Phi$ is positive. From the positivity of the operators $\mathcal{Q}_{\mu}$ and $\Phi$ it follows that operator (54) is also positive in $C$ for every sufficiently large $\mu$, which completes the proof.

\subsection{Proof of Lemma 2}

We write $w_{n} \stackrel{\mathrm{w}}{\rightarrow} w_{*}$ for the weak convergence in $L^{2}$.

First note that the completely continuous operator $B_{\nu}^{(1 / 2)}: L^{2} \rightarrow C$ maps any weakly converging sequence $w_{n} \in L^{2}, \quad w_{n} \stackrel{\mathrm{w}}{\rightarrow} w_{*}$ to the sequence $B_{\nu}^{(1 / 2)} w_{n} \in C$ that converges uniformly to the function $B_{\nu}^{(1 / 2)} w_{*}$. Therefore functional (37) is weakly continuous, which means that $w_{n} \stackrel{\text { w }}{\rightarrow} w_{*}$ implies $\mathcal{F}_{\alpha}\left(w_{n}\right) \rightarrow \mathcal{F}_{\alpha}\left(w_{*}\right)$. The same is true for the quadratic functional $\left(w, B_{\nu} w\right)_{L^{2}}=\left\|B_{\nu}^{(1 / 2)} w\right\|_{L^{2}}^{2}$. At the same time, the functional $(w, w)_{L^{2}}$ is weakly lower semicontinuous, i.e.,

$$
w_{n} \stackrel{\mathrm{w}}{\rightarrow} w_{*} \quad \text { implies } \quad \lim _{n} \inf \left(w_{n}, w_{n}\right)_{L^{2}} \geq\left(w_{*}, w_{*}\right)_{L^{2}} .
$$

Therefore the functional $V_{\alpha}(w)=(1 / 2)(w, w)_{L^{2}}-(\nu / 2)\left(w, B_{\nu} w\right)_{L^{2}}-$ $\mathcal{F}_{\alpha}(w)$ is also weakly lower semicontinuous:

$$
w_{n} \stackrel{\mathrm{w}}{\rightarrow} w_{*} \quad \text { implies } \quad \lim _{n} \inf V_{\alpha}\left(w_{n}\right) \geq V_{\alpha}\left(w_{*}\right) .
$$

By definition $\bar{G}(t, x, \alpha) \leq G(t, P x)$ for all $t, x$. Since estimate (2) holds for all $x \in K_{+}$and we have $|P x| \leq|x|$ and $P x \in K_{+}$for each $x \in R^{N}$, the relations

$$
\bar{G}(t, x, \alpha) \leq G(t, P x) \leq \kappa|P x|^{2}+b \leq \kappa|x|^{2}+b, \quad x \in R^{N}
$$

\footnotetext{
${ }^{11}$ In fact, the series also converges in C, because $\left\|\mathcal{Q}_{\mu}\right\|_{L^{2} \rightarrow C}<\infty$.
} 
are valid. Therefore

$$
\begin{aligned}
\mathcal{F}_{\alpha}(w) & \leq \kappa \int_{0}^{1}\left|B_{\nu}^{(1 / 2)} w(t)\right|^{2} d t+b \\
& =\kappa\left(B_{\nu}^{(1 / 2)} w, B_{\nu}^{(1 / 2)} w\right)_{L^{2}}+b=-\frac{\nu}{2}\left(w, B_{\nu} w\right)_{L^{2}}+b
\end{aligned}
$$

Hence, functional (34) satisfies the estimate

$$
V_{\alpha}(w) \geq \frac{1}{2}(w, w)_{L^{2}}-b, \quad w \in L^{2} .
$$

Consider a minimizing sequence $w_{n}$ for the functional (34):

$$
\lim _{n} V_{\alpha}\left(w_{n}\right)=\inf _{w \in L^{2}} V_{\alpha}(w)
$$

The equality $V_{\alpha}(0)=0$ implies $0 \geq \lim V_{\alpha}\left(w_{n}\right)$. But from (57) it follows that $V_{\alpha}(w) \geq \varepsilon^{2} / 2$ whenever $\|w\|_{L^{2}} \geq \sqrt{2 b}+\varepsilon$, therefore

$$
\lim _{n} \sup \left\|w_{n}\right\|_{L^{2}} \leq \sqrt{2 b}
$$

This implies $\sup \left\{\left\|w_{n}\right\|_{L^{2}}: n=1,2, \ldots\right\}<\infty$, hence the sequence $w_{n}$ is weakly compact and contains a weakly converging subsequence. Without loss of generality, assume that the sequence $w_{n}$ weakly converges itself and denote its limit by $w_{\alpha}^{*}$. From (56) the relations

$$
\inf _{w \in L^{2}} V_{\alpha}(w)=\lim _{n} V_{\alpha}\left(w_{n}\right) \geq V_{\alpha}\left(w_{\alpha}^{*}\right)
$$

follow, so $V_{\alpha}\left(w_{\alpha}^{*}\right)=\inf _{w \in L^{2}} V_{\alpha}(w)$, i.e., $w_{\alpha}^{*}$ is a point of global minimum for the functional $V_{\alpha}(w)$. From (55) and (58) estimate (42) follows. To prove the inclusion ${ }^{12} w_{\alpha}^{*} \in \mathcal{E}$, we use an idea close to [13].

Fix any positive function $y(t) \in C^{2}$ such that

$$
y(0)=y(1)=0, \quad y_{i}(t)>0 \quad \text { for all } t \in(0,1), i=1, \ldots, N
$$

\footnotetext{
${ }^{12}$ In fact, the inclusion $w_{\alpha}^{*} \in \mathcal{E}$ means that the function $x_{\alpha}(t)=B_{\nu}^{(1 / 2)} w_{\alpha}^{*}(t)$ is a socalled proper solution of problem (28). The notion of a proper solution was introduced in [12].
} 
and put $h(t):=B_{\nu}^{(1 / 2)}\left(-y^{\prime \prime}(t)+A(t) y(t)+\nu y(t)\right)$, then $y(t)=B_{\nu}^{(1 / 2)} h(t)$. Consider the function

$$
\Psi(\theta)=V_{\alpha}\left(w_{\alpha}^{*}+\theta h\right)+V_{\alpha}\left(w_{\alpha}^{*}-\theta h\right)-2 V_{\alpha}\left(w_{\alpha}^{*}\right), \quad \theta \in[0,1] .
$$

Since $w_{\alpha}^{*}$ is a minimum point for $V_{\alpha}(w)$, we have $\Psi(\theta) \geq 0$ for each $\theta \in[0,1]$. But (39) implies the equality

$$
\mathcal{F}_{\alpha}\left(w_{\alpha}^{*}+\theta h\right)+\mathcal{F}_{\alpha}\left(w_{\alpha}^{*}-\theta h\right)-2 \mathcal{F}_{\alpha}\left(w_{\alpha}^{*}\right)=\theta \psi(\theta)
$$

where

$$
\begin{array}{r}
\psi(\theta):=\int_{0}^{1} \int_{0}^{1}\left(y(t), \bar{g}\left(t, x_{\alpha}(t)+\theta s y(t) ; \alpha\right)\right. \\
\left.-\bar{g}\left(t, x_{\alpha}(t)-\theta s y(t) ; \alpha\right)\right) d s d t
\end{array}
$$

and $x_{\alpha}(t)=B_{\nu}^{(1 / 2)} w_{\alpha}^{*}(t)$. Hence,

$$
\Psi(\theta)=\theta^{2}\left(h,\left(I-\nu B_{\nu}\right) h\right)_{L^{2}}-\theta \psi(\theta), \quad \theta \in[0,1]
$$

and the estimate $\Psi(\theta) \geq 0$ implies the relation

$$
\lim _{\theta \rightarrow+0} \sup \psi(\theta) \leq 0 .
$$

Set

$$
\Pi_{t}^{i}=\left\{x=\left(x_{1}, \ldots, x_{N}\right)^{T}: x_{i}=0, g_{i}(t, P x) \neq 0\right\} .
$$

By definition, the function $\bar{g}_{i}(t, x ; \alpha)$ is continuous in $x$ at every point $x^{0} \in R^{N} \backslash \Pi_{t}^{i}$, hence

$$
\lim _{\theta \rightarrow+0}\left(\bar{g}_{i}\left(t, x^{0}+\theta z ; \alpha\right)-\bar{g}_{i}\left(t, x^{0}-\theta z ; \alpha\right)\right)=0
$$

for each $x^{0} \in R^{N} \backslash \Pi_{t}^{i}, z \in R^{N}$. If $x^{0} \in \Pi_{t}^{i}$, then $x_{i}^{0}=0$ and (27) implies

$$
\begin{gathered}
\lim _{\theta \rightarrow+0}\left(\bar{g}_{i}\left(t, x^{0}+\theta z ; \alpha\right)-\bar{g}_{i}\left(t, x^{0}-\theta z ; \alpha\right)\right)=\operatorname{sign} z_{i} \cdot g_{i}\left(t, P x^{0}\right) \\
\text { for each } x^{0} \in \Pi_{i}^{i}, z \in R^{N} .
\end{gathered}
$$

Thus, passing to the limit in (60) as $\theta \rightarrow+0$ and taking into account that $s y_{i}(t)>0$ for every $t, s \in(0,1)$, we obtain

$$
\psi(+0)=\sum_{i=1}^{N} \int_{\Omega_{i}} y_{i}(t) g_{i}\left(t, P x_{\alpha}(t)\right) d t
$$


where $\Omega_{i}:=\left\{t \in[0,1]: x_{\alpha}(t) \in \Pi_{t}^{i}\right\}$, so according to (61),

$$
\sum_{i=1}^{N} \int_{\Omega_{i}} y_{i}(t) g_{i}\left(t, P x_{\alpha}(t)\right) d t \leq 0 .
$$

But from (8) it follows that $g_{i}(t, P x) \geq 0$ whenever $x_{i}=0, x=$ $\left(x_{1}, \ldots, x_{N}\right)^{T}$, hence $g_{i}(t, P x)>0$ for each $x \in \Pi_{t}^{i}$ and therefore, $g_{i}\left(t, P x_{\alpha}(t)\right)>0$ for all $t \in \Omega_{i}, i=1, \ldots, N$. Together with (59) and (62), this implies mes $\Omega_{i}=0, i=1, \ldots, N$, which is equivalent to the inclusion $w_{\alpha}^{*} \in \mathcal{E}$.

It remains to show that under the conditions of Theorem 3 the relation $w_{\alpha}^{*} \neq 0$ is valid, i.e., zero is not a global minimum point for the functional $V_{\alpha}(w)$. We prove that zero is not even a local minimum point. For this, an appropriate element $h^{0} \in L^{2}$ is introduced so that $V_{\alpha}\left(\theta h^{0}\right)<V_{\alpha}(0)$ for each small $\theta \neq 0$.

Denote by $T_{\mu}, \mu \neq-\mu_{n}, n=0,1,2, \ldots$, the operator that maps any function $u(t) \in C$ to the unique solution $x(t)=T_{\mu} u(t) \in C^{2}$ of the problem $\mathcal{M} x(t)+\mu x(t)=u(t)$ where $\mathcal{M}$ is operator (11). Since $g(t, 0) \equiv 0$, it follows from (8) that

$$
\frac{\partial g_{i}}{\partial x_{j}}(t, 0) \geq 0, \quad i \neq j, t \in[0,1],
$$

hence the off-diagonal elements of the symmetric matrix $A(t)-D(t)$ satisfy the estimate

$$
a_{i j}(t)-d_{i j}(t) \leq 0 \text { for all } i \neq j, i, j=1, \ldots, N ; t \in[0,1],
$$

similar to (4). By Lemma 3, this estimate implies that for every sufficiently large $\mu>-\mu_{0}$ the operator $T_{\mu}: C \rightarrow C$ is positive w.r.t. the cone of positive functions. Also, $T_{\mu}$ is completely continuous, so, according to the general theorems on positive operators [8], there is a positive eigenvector $y^{0}=y^{0}(t) \geqslant 0$ such that $T_{\mu} y^{0}=r\left(T_{\mu}\right) y^{0}$ where $r\left(T_{\mu}\right)$ is the spectral radius of the operator $T_{\mu}$. For large $\mu$ we have $r\left(T_{\mu}\right)=\left(\mu+\mu_{0}\right)^{-1}$, therefore $y^{0}(t)$ is a classical nonzero positive solution of the problem $\mathcal{M} y(t)+\mu y(t)=\left(\mu+\mu_{0}\right) y(t)$, i.e., the identity $\left(\mathcal{M}-\mu_{0} I\right) y^{0}(t)=0$ holds.

Set

$$
\nu^{0}(t)=\left(\nu+\mu_{0}\right) y^{0}(t)+D(t) y^{0}(t), \quad h^{0}(t)=B_{\nu}^{\frac{1}{2}} \nu^{0}(t)
$$


Adding the function $v^{0}(t)$ to the identity $\left(\mathcal{M}-\mu_{0} I\right) y^{0}(t)=0$, we get $(\mathcal{L}+\nu I) y^{0}(t)=v^{0}(t)$, therefore $y^{0}(t)=B_{\nu} v^{0}(t)$ and $y^{0}(t)=B_{\nu}^{(1 / 2)} h^{0}(t)$. Consider the function $\chi(\theta):=V_{\alpha}\left(\theta h^{0}\right)$. Since $\bar{G}(t, x ; \alpha)=G(t, x)$ for each $x \geq 0$ and $y^{0}(t)=B_{\nu}^{(1 / 2)} h^{0}(t) \geq 0$, we have

$$
V_{\alpha}\left(\theta h^{0}\right)=\frac{\theta^{2}}{2}\left(h^{0}, h^{0}\right)_{L^{2}}-\frac{\nu \theta^{2}}{2}\left(h^{0}, B_{\nu} h^{0}\right)_{L^{2}}-\int_{0}^{1} G\left(t, \theta y^{0}(t)\right) d t .
$$

Our assumptions on the smoothness of function (7) imply the representation

$$
G(t, x)=G(t, 0)+(g(t, 0), x)+(x, D(t) x) / 2+\varphi(t, x)
$$

where

$$
\lim _{x \rightarrow 0}|x|^{-2} \max _{t \in[0,1]}|\varphi(t, x)|=0 .
$$

But $G(t, 0) \equiv g(t, 0) \equiv 0$, so $G(t, x)=(x, D(t) x) / 2+\varphi(t, x)$ and therefore

$$
\begin{gathered}
V_{\alpha}\left(\theta h^{0}\right)=\frac{\theta^{2}}{2}\left(\left(h^{0}, h^{0}\right)_{L^{2}}-\nu\left(h^{0}, B_{\nu} h^{0}\right)_{L^{2}}-\left(y^{0}(t), D(t) y^{0}(t)\right)_{L^{2}}\right)+o\left(\theta^{2}\right), \\
\theta \rightarrow 0 .
\end{gathered}
$$

Now note that $\left(h^{0}, B_{\nu} h^{0}\right)_{L^{2}}=\left(y^{0}, y^{0}\right)_{L^{2}}$ and

$$
\begin{aligned}
\left(h^{0}, h^{0}\right)_{L^{2}} & =\left(v^{0}, B_{\nu} v^{0}\right)_{L^{2}}=\left(v^{0}, y^{0}\right)_{L^{2}} \\
& =\left(\left(\nu+\mu_{0}\right) y^{0}(t)+D(t) y^{0}(t), y^{0}(t)\right)_{L^{2}},
\end{aligned}
$$

hence $V_{\alpha}\left(\theta h^{0}\right)=\mu_{0} \theta^{2}\left(y^{0}, y^{0}\right)_{L^{2}} / 2+o\left(\theta^{2}\right)$. By assumption, $\mu_{0}<0$, so $V_{\alpha}\left(\theta h^{0}\right)<0=V_{\alpha}(0)$ for all small $\theta \neq 0$, i.e., zero is not a minimum point for the functional $V_{\alpha}(w)$ and the proof of Lemma 2 is complete.

\section{PROOF OF THEOREMS 5 AND 6}

\subsection{Proof of Lemma 1}

Define the nonlinear operator

$$
U_{\lambda} x(t)=B_{\lambda}(f(t, x(t))+\lambda x(t)), \quad x(t) \in C
$$


with $\lambda \neq \lambda_{n}, n=0,1,2, \ldots$ Now problem (12) is equivalent to the equation $x(t)=U_{\lambda} x(t)$. We need to show that this equation has a solution in the $\operatorname{set}^{13}$

$$
\left\langle x_{-}, x_{+}\right\rangle=\left\{x(t) \in C: x_{-}(t) \leq x(t) \leq x_{+}(t)\right\}
$$

where $x_{-}(t)$ and $x_{+}(t)$ are solutions of problems (15), i.e., they satisfy the relations

$$
x_{-}(t)=B_{\lambda}\left(h\left(t, x_{-}(t)\right)+\lambda x_{-}(t)\right), \quad x_{+}(t)=B_{\lambda}\left(g\left(t, x_{+}(t)\right)+\lambda x_{+}(t)\right) .
$$

Since $U_{\lambda}$ is a completely continuous operator in $C$, it suffices to prove $U_{\lambda}$ maps the convex closed set $\left\langle x_{-}, x_{+}\right\rangle$into itself, then the existence of a solution $x^{*}=x^{*}(t)$ to equation $x=U_{\lambda} x$ follows from the Schauder principle.

Suppose $x(t) \in\left\langle x_{-}, x_{+}\right\rangle$; then by assumption,

$$
h(t, x(t)) \leq f(t, x(t)) \leq g(t, x(t)) .
$$

Since the components $h_{i}\left(t, x_{i}\right), g_{i}\left(t, x_{i}\right)$ of the functions $h(t, x)$ and $g(t, x)$ are smooth, for every $r>0$ there is a $\lambda(r)$ such that the functions $h_{i}\left(t, x_{i}\right)+\lambda x_{i}$ and $g_{i}\left(t, x_{i}\right)+\lambda x_{i}$ increase w.r.t. $x_{i}$ in the segment $-r \leq x_{i} \leq r$ for every $\lambda \geq \lambda(r)$ and every $t \in[0,1], i=1, \ldots, N$. Therefore,

$$
\begin{aligned}
h\left(t, x_{-}(t)\right)+\lambda x_{-}(t) & \leq h(t, x(t))+\lambda x(t) \\
g(t, x(t))+\lambda x(t) & \leq g\left(t, x_{+}(t)\right)+\lambda x_{+}(t)
\end{aligned}
$$

whenever $x(t) \in\left\langle x_{-}, x_{+}\right\rangle$and $\lambda \geq \lambda\left(r_{0}\right)$ with $r_{0}:=\max \left\{\left\|x_{-}\right\|_{C}\right.$, $\left.\left\|x_{+}\right\|_{+}\right\}$. Combined with (63), this implies the estimates

$$
h\left(t, x_{-}(t)\right)+\lambda x_{-}(t) \leq f(t, x(t))+\lambda x(t) \leq g\left(t, x_{+}(t)\right)+\lambda x_{+}(t) .
$$

If $\lambda$ is sufficiently large, then by Lemma 3 the operator $B_{\lambda}$ is positive, so applying this operator to (64), one obtains

$$
B_{\lambda}\left(h\left(t, x_{-}(t)\right)+\lambda x_{-}(t)\right) \leq U_{\lambda} x(t) \leq B_{\lambda}\left(g\left(t, x_{+}(t)\right)+\lambda x_{+}(t)\right)
$$

or, which is the same, $x_{-}(t) \leqslant x(t) \leqslant x_{+}(t)$. That is, $x(t) \in\left\langle x_{-}, x_{+}\right\rangle$ implies $U_{\lambda} x(t) \in\left\langle x_{-}, x_{+}\right\rangle$and the proof is complete.

\footnotetext{
${ }^{13}$ This set is the so-called cone interval with the vertexes $x_{-}, x_{+}$.
} 


\subsection{Proof of Theorems 5 and 6}

Suppose the conditions of Theorem 5 hold. Set $g(t, x)=\left(g_{1}\left(t, x_{1}\right)\right.$, $\left.\ldots, g_{N}\left(t, x_{N}\right)\right)^{T}$ and consider the problem

$$
-x^{\prime \prime}+A(t) x=g(t, x), \quad x(0)=x(1)=0 .
$$

Relation (17) implies that problem (65) is equivalent to the $k$ independent two-point problems in the subspaces $R^{N_{i}-N_{i-1}}, i=1, \ldots, k$ of $R^{N}$. Evidently, each of the smaller problems satisfies all the assumptions of Theorem 2 , hence there is a positive solution $z_{i}^{*}(t)$ : $[0,1] \rightarrow R^{N_{i}-N_{i-1}}$ to each smaller problem, so the direct sum of these solutions is a positive solution $x_{+}(t):[0,1] \rightarrow R^{N}$ of problem (65).

Assume that in place of (18) the stronger estimate

$$
f_{j}(t, x) \geq \varepsilon \quad \text { whenever } \quad x_{j}=0, x \in K_{+}
$$

holds with some $\varepsilon>0$ for each $j=1, \ldots, N$. In this case, we can find a large $\gamma$ such that

$$
-\gamma x_{j} \leq f_{j}(t, x) \text { for all } 0 \leq x_{1}, \ldots, x_{N} \leq R:=\left\|x_{+}(t)\right\|_{C}
$$

and all $t \in[0,1], j=1, \ldots, N$. Now, if we take $h(t, x)=-\gamma x, x_{-}(t) \equiv 0$, then all the conditions of Lemma 1 are satisfied, hence problem (12) has a solution $0 \leqslant x^{*}(t) \leqslant x_{+}(t)$. Thus, the conclusion of Theorem 5 is true if additional assumption (66) holds.

To complete the proof, one can first replace the functions $f(t, x)$ and $g(t, x)$ in (12) and (65) by the functions

$$
\begin{aligned}
& f(t, x ; \varepsilon):=\left(f_{1}(t, x)+\varepsilon, \ldots, f_{N}(t, x)+\varepsilon\right)^{T} \\
& g(t, x ; \varepsilon):=\left(g_{1}\left(t, x_{1}\right)+\varepsilon, \ldots, g_{N}\left(t, x_{N}\right)+\varepsilon\right)^{T}
\end{aligned}
$$

and prove just as above the existence of a positive solution $x_{\varepsilon}(t)$ to the problem

$$
-x^{\prime \prime}+A(t) x=f(t, x ; \varepsilon), \quad x(0)=x(1)=0 .
$$

Then the same limit construction as used in the proof of Theorem 1 can be applied to obtain a positive solution of problem (12) (we omit the further details).

Finally, let us prove Theorem 6. By assumption (20), at least one of the numbers $\mu_{0 i}$ is negative, without loss of generality suppose that 
$\mu_{01}<0$. Consider the problem

$$
-z^{\prime \prime}+A_{1}(t) z=\tilde{h}(t, z), \quad z(0)=z(1)=0
$$

where $z \in R^{N_{1}}, \tilde{h}(t, z)=\left(h_{1}\left(t, z_{1}\right), \ldots, h_{N_{1}}\left(t, z_{N_{1}}\right)\right)^{T}$. Since $h_{j}(t, \xi) \leq$ $g_{j}(t, \xi)$ for all $\xi \geq 0, j=1, \ldots, N$, estimate (19) implies that

$$
\sum_{j=1}^{N_{1}} \int_{0}^{z_{j}} h_{j}(t, \xi) d \xi \leq \kappa_{1} \sum_{j=1}^{N_{1}} z_{j}^{2}+b_{1}, \quad z_{1}, \ldots, z_{N_{1}} \geq 0 .
$$

Therefore problem (68) satisfies all the conditions of Theorem 4, so it has a nonzero solution $z^{*}(t) \geqslant 0$ and hence the function $x_{-}(t)=$ $\left(z_{1}^{*}(t), \ldots, z_{N_{1}}^{*}(t), 0, \ldots, 0\right)^{T}$ is a nonzero positive solution to the first problem of (15). Now it suffices to show that the second problem of (15) has a solution $x_{+}(t)=x_{-}(t)+y(t)$ with $y(t) \geqslant 0$ and to use Lemma 1. After the change of the variable $x=y+x_{-}(t)$, the second problem of (15) takes the form

$$
-y^{\prime \prime}+A(t) y=\hat{g}(t, y), \quad y(0)=y(1)=0
$$

where

$$
\begin{aligned}
\hat{g}(t, y) & =g\left(t, x_{-}(t)+y\right)+\left(x_{-}^{\prime \prime}(t)-A(t) x_{-}(t)\right) \\
& =g\left(t, x_{-}(t)+y\right)-h\left(t, x_{-}(t)\right) .
\end{aligned}
$$

First note that $x_{-}(t) \geqslant 0$ implies $\hat{g}(t, 0)=g\left(t, x_{-}(t)\right)-h\left(t, x_{-}(t)\right) \geq$ 0 , i.e., the function $\hat{g}(t, 0)$ is positive. Secondly, from estimates (19) it follows that for any number $q_{i} \in\left(\kappa_{i}, \lambda_{0 i}\right)$ there is a sufficiently large $\beta_{i}>0$ such that the components of $\hat{g}(t, y)$ satisfy

$$
\begin{aligned}
& \sum_{j=N_{i-1}+1}^{N_{i}} \int_{0}^{y_{j}} \hat{g}_{j}(t, \xi) d \xi \leq q_{i} \sum_{j=N_{i-1}+1}^{N_{i}} y_{j}^{2}+\beta_{i} \\
& \text { whenever } \quad y_{N_{i-1}+1} \geq 0, \ldots, y_{N_{i}} \geq 0
\end{aligned}
$$

for each $i=1, \ldots, k$. Splitting problem (69) according to (17) into the $k$ independent problems in the subspaces $R^{N_{i}-N_{i-1}}$ of $R^{N}$, we see that each of the $k$ problems satisfies all the assumptions of Theorem 2 and so has a positive solution. The direct sum $y^{*}(t)$ of these solutions is a positive solution of problem (69). It determines the solution $x_{+}(t)=x_{-}(t)+y^{*}(t) \geqslant x_{-}(t)$ to the second problem of (15) and by 
Lemma 1, problem (12) has a solution $x^{*}(t) \in\left\langle x_{-}, x_{+}\right\rangle$. The relations $x_{-}(t) \geqslant 0, x_{-}(t) \not \equiv 0$ imply that $x^{*}(t)$ is the desired nonzero positive solution of (12). Theorem 6 is proved.

\section{Acknowledgement}

The research was done while D. Rachinskii was visiting the Regensburg University on the basis of a Research Fellowship from the Alexander von Humboldt Foundation.

\section{References}

[1] Krasnosel'skii, M. A., Topological Methods in the Theory of Nonlinear Integral Equations, Pergamon Press, Oxford, 1964.

[2] Krasnosel'skii, M. A. and Zabreiko, P. P., Geometrical Methods of Nonlinear Analysis, Springer-Verlag, Berlin, 1984.

[3] Vainberg, M. M., Variational Method and Method of Monotone Operators, Halsted Press, New York, 1973.

[4] Hammerstein, A. (1930). Nichtlineare Integralgleichungen nebst Anwendungen, Acta Math., 54, 117-176.

[5] Golomb, M. (1935). Zur Theorie der nichtlinearen Integralgleichungen, Integralgleichungssysteme und allgemeinen Funktionalgleichungen, Math. Zeitschrift, 39, 45- 75 .

[6] Krein, M. G. and Rutman, M. A. (1950). Linear operators leaving invariant a cone in a Banach space, Amer. Math. Soc. Translation, 26.

[7] Krasnosel'skii, M. A., Positive Solutions of Operator Equations, Noordhoff, Groningen, 1964.

[8] Krasnosel'skii, M. A., Lifshitz, E. A. and Sobolev, V. A., Linear Positive Systems: The Method of Positive Operators, Hilderman Verlag, Berlin, 1990.

[9] Atkinson, F. V., Discrete and Continuous Boundary Problems, Academic Press, New York, 1964.

[10] Krasnosel'skii, M. A., Mennicken, R. and Rachinskii, D. I. (1997). Potential bounds in nonpotential two-point boundary-value problems, Math. Nachr., 188, $203-218$.

[11] Krasnosel'skii, M. A., Zabreiko, P. P., Pustylnik, E. I. and Sobolevskii, P. E., Integral Operators in Spaces of Summable Functions, Noordhoff, Leyden, 1976.

[12] Krasnosel'skii, M. A. and Pokrovskii, A. V. (1979). Regular solutions of equations with discontinuous nonlinearities, Soviet Math. Dokl., 17(1), 128-132.

[13] Pavlenko, V. N. (1988). Existence theorems for elliptic variational inequalities with quasipotential operators, Differential Equations, 24(8), 913-916. 